\title{
Pupil autoregulation impairment as an early marker of glaucomatous damage
}

\author{
Marta Anna Szmigiel ${ }^{1, A-F}$, Joanna Wiktoria Przeździecka-Dołyk1,2,A-F, Jacek Olszewski, ${ }^{1, C, E, F}$, Henryk Kasprzak ${ }^{1, A, C-F}$ \\ ${ }^{1}$ Department of Optics and Photonics, Faculty of Fundamental Problems of Technology, Wroclaw University of Science and Technology, Poland \\ ${ }^{2}$ Department and Clinic of Ophthalmology, Wroclaw Medical University, Poland \\ A - research concept and design; $\mathrm{B}$ - collection and/or assembly of data; $\mathrm{C}$ - data analysis and interpretation; \\ $D$ - writing the article; $E$ - critical revision of the article; $F$ - final approval of the article
}

\section{Address for correspondence \\ Marta Szmigiel \\ E-mail: marta.szmigiel@pwr.edu.pl}

\section{Funding sources}

M. Szmigiel acknowledges that part of this work was supported by the National Science Centre conferred on the basis of decision No. DEC-2013/11/N/ST7/00366.

\section{Conflict of interest}

None declared

Received on 0ctober 14, 2018

Reviewed on January 24, 2019

Accepted on May 14, 2019

Published online on September 11, 2019

Cite as

Szmigiel MA, Przé́dziecka-Dołyk JW, Olszewski J,

Kasprzak H. Pupil autoregulation impairment as an early marker of glaucomatous damage. Adv Clin Exp Med. 2019;28(10):1367-1375. doi:10.17219/acem/109343

DOI

10.17219/acem/109343

\section{Copyright}

Copyright by Author(s)

This is an article distributed under the terms of the

Creative Commons Attribution Non-Commercial License

(http://creativecommons.org/licenses/by-nc-nd/4.0/)

\begin{abstract}
Background. Glaucoma, a degenerative and progressive disease, leads to structural and functional changes in the optic nerve head and retinal ganglion cells (RGCS), while the vasculature of the iris stays intact.
\end{abstract}

Objectives. The aim of this study was to determine whether the coherence level associated with pupil geometry and peripheral arterial pulsation can be the basis for differentiating glaucoma and glaucomasuspected patients from a control group.

Material and methods. This is an investigator-initiated, single-center prospective cohort study. Patients with diagnosed glaucoma (glaucoma group - GG) or glaucoma suspects (glaucoma suspects group - GSG), as well as healthy participants (control group - (G), were prospectively enrolled in the study. Glaucomadiagnosed patients and glaucoma suspects who converted to glaucoma within 5 years were included. All patients underwent a full ophthalmological examination that included measurements of the thicknesses of the retinal nerve fiber layer (RNFL) and the ganglion cell complex (GCC) along with other parameters. A custom-made pupilometer was synchronized with a pulsometer to simultaneously record an image of the pupil and the peripheral arterial pulsation signal. All readings were processed with a script developed by the researchers. The main indicator of an increased influence of the vascular structures of the iris on pupil geometry in the patients and $(G$ were the coherence levels (levC) between parameters describing the pupillary shape and peripheral arterial pulsation.

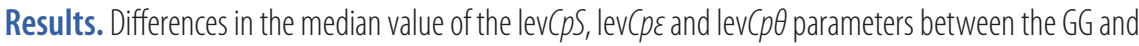
GSG compared to the CG were found $(p<0.001)$. During the follow-up period, a larger decrease was observed in RNFL thickness and GCC thickness in the GSG than in the GG $(p<0.05)$. Strong correlations were found between levC $p S$ and RNFL and GCC loss among the GSG group ( $p<0.001)$, while in the GG this parameter correlated with RNFL and GCC thickness $(p<0.001)$.

Conclusions. This is the first attempt to relate changes in the neuronal signaling pathways in glaucoma to the vascular-dependent changes of pupil geometry. The findings presented herein suggest that this approach can be used to determine which glaucoma suspects have autonomic system impairment in the eye, increasing their probability of glaucoma conversion.

Key words: glaucoma, retinal nerve fiber layer, pupil, ganglion cell complex, peripheral arterial pulsation 


\section{Introduction}

Glaucoma is the most common type of optic neuropathy. According to the World Health Organization (WHO), it represents one of the leading causes of irreversible blindness in developed countries, along with age-related macular degeneration and diabetic retinopathy. ${ }^{1,2}$ The pathomechanism of glaucomatous optic neuropathy has not been fully explained. This degenerative and progressive disease leads to structural and functional changes in both the optic nerve head and in the retinal ganglion cells (RGCs).

Although the majority of RGCs are involved in cortical image processing and recognition, there is a small group of RGCs that project the lateral geniculate nucleus, the pretectal olivary nucleus and the suprachiasmatic nucleus. This non-image-forming pathway (Fig. 1A) is managed by a different type of RGCs, called intrinsically photosensitive RGCs (ipRGCs), comprising approx. $0.8-3 \%$ of the total RGC population in the human retina. Assuming that

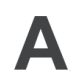

A

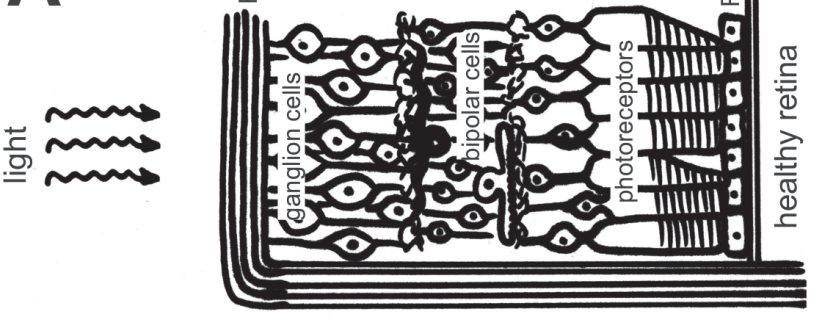

optic nerve
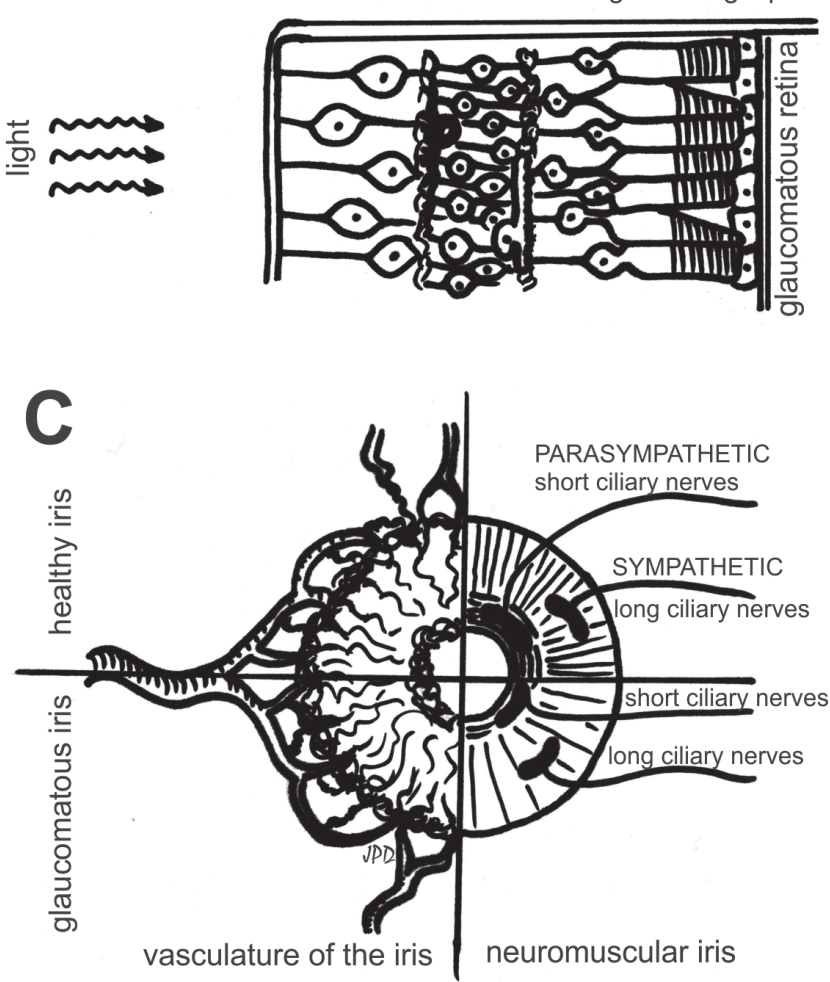

the total number of RGCs in the human retina is approx. 1.2 million, only 9.6-36 thousand RGCs contain melanopsin and are intrinsically photosensitive. ${ }^{3-5}$ In a healthy retina, a light impulse - after being transduced into a biochemical impulse - is transmitted via the cortical and nonimage-forming pathways towards the appropriate centers in the brain. However, in the glaucomatous retina, the same light impulse causes less pronounced cortical and nonimage-forming input towards the brain due to the presence of fewer RGCs in the retina (Fig. 1A). Non-image forming input is transmitted through the optic tract to the pretectal nucleus, where it acts as an excitatory impulse for the Edinger-Westphal nucleus. The impulses from this nucleus travel through the oculomotor nerve (its parasympathetic part) to the ciliary ganglion and, after switching to the postsynaptic neuron, as short ciliary nerves to the iris. Arousal of the sympathetic part of reticular formation results in inhibitory impulses that travel from the hypothalamus to the Edinger-Westphal nucleus. Simultaneously, arousal

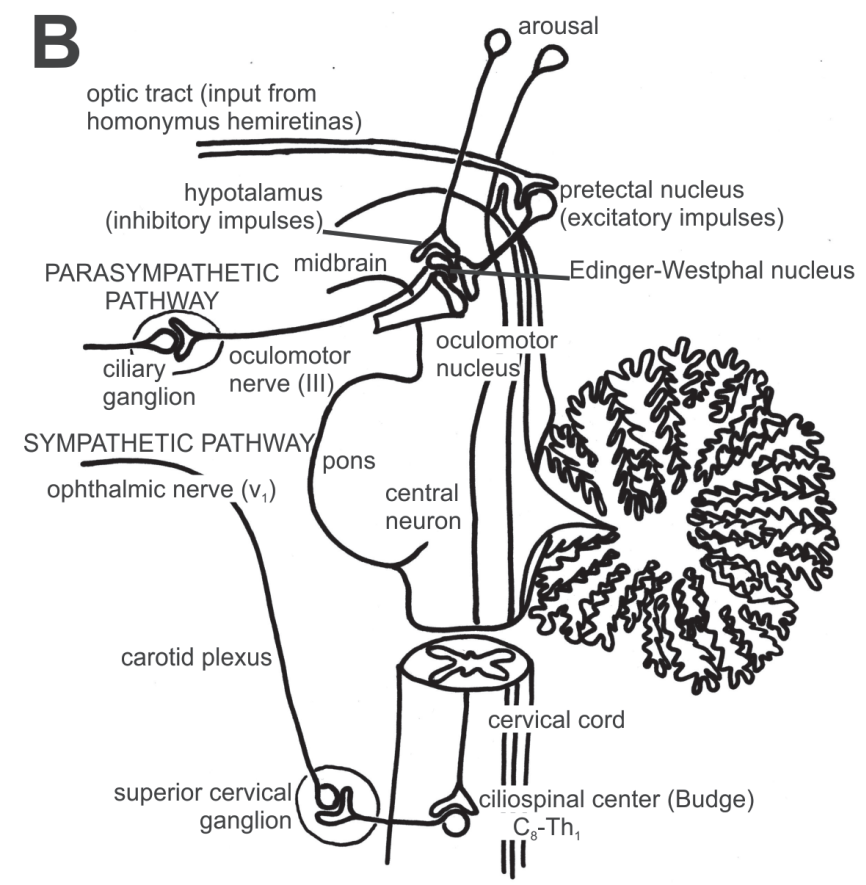

Fig. 1. Healthy (top) and glaucomatous (bottom) retina with important cells for cortical image and non-image forming input (A), non-image forming pathways in brain and spinal cord (B) and healthy (top) and glaucomatous (bottom) iris with vascular (left) and neuromuscular (right) (C) anatomic features 
of the sympathetic part of the reticular formation is transmitted through the cervical cord to the ciliospinal center located in the $\mathrm{C}_{8}-\mathrm{Th}_{1}$. The sympathetic presynaptic nerve leaves the spinal cord and forms synapses in the superior cervical ganglion. The postsynaptic sympathetic nerves form the carotid plexus and travel upward with this vessel, then along with the ophthalmic nerve $\left(V_{1}\right)$ they enter the tissues of the eye as long ciliary nerves (Fig. 1B).

In the iris as well, there are differences between glaucomatous and normal neuromuscular structures. The reduced input from RGCs in glaucomatous patients results in muscle atrophy due to insufficient impulse creation when compared to a healthy eye, but the vasculatures of glaucomatous and healthy irises are similar. The peripheral arterial pulsation, while invisible in a healthy eye, becomes pronounced in a glaucomatous one. This provides the background for our study (Fig. 1C).

In 2002, Hattar et al. and Berson et al. simultaneously described a new type of photoreceptor that expresses the photopigment melanopsin (also known as opsin 4 or OPN4). ${ }^{6,7}$ In recent years, the investigators have shown that light transmission to the hypothalamic suprachiasmatic nucleus or the post-illumination pupillary light response (PLR) is compromised as a result of ipRGC damage in glaucoma. Gracitelli et al. showed that there is a positive association between ipRGCs and retinal nerve fiber layer (RNFL) thinning in glaucoma. In addition, it has also been proven that the severity of glaucoma reflected by a standard automated perimeter mean deviation worse than $12 \mathrm{~dB}$ was associated with a worse pupillary response. ${ }^{5,8,9}$ The correlation between ipRGCs and RNFL is represented in Fig. 1A, demonstrating a significant reduction in the number of RGCs and in RNFL thickness in a glaucomatous retina. Kuze et al. suggested that electroretinography (ERG) may be a promising method in treating a variety of pathological conditions in which ipRGC disorders are possible. In that study, glaucomatous patients showed diminished responses of the ipRGCs in ERG. ${ }^{4}$ The reduced blue-red post-illumination pupil response indicates a characteristic impairment of the melanopsin-driven pathway of ipRGCs in patients with glaucoma. Other studies have shown that reduced maximal relative amplitude and increased slope of the response during exposure to the 4-second red stimulus suggest a disturbed synaptic function and an altered interaction between outer photoreceptors, RGCs and ipRGCs. ${ }^{10,11}$ In glaucomatous patients, the diminished input from hemiretinas results in lower excitatory impulses being projected from the pretectal nucleus to the Westphal-Edinger nucleus (Fig. 1B). This results in a decrease of the parasympathetic pathway activity transmitted to the iris muscles which contract the pupil (Fig. 1B,C).

Detection of the pupil edge is used to determine the parameters that describe the pupil and its changes over time in an unequivocal and comparable manner. Pupil geometry is quite complex ${ }^{12}$ and is characterized by rapid changes in its geometrical parameters over time, ${ }^{13}$ with a constant level of light and constant conditions of its stimulation. For various lighting conditions, the pupil can grow in area even several times larger. Normal pupil diameter in bright light varies in adults from $2 \mathrm{~mm}$ to $4 \mathrm{~mm}$ and in dim light from $4 \mathrm{~mm}$ to $8 \mathrm{~mm}$. Even with constant lighting, the shape and size of the pupil are variable. Measurements of pupil diameter after dark adaptation and during pulsing light stimulation are presented in a study by Gooley et al. ${ }^{14}$ In a paper by Winn et al., ${ }^{15}$ the authors measured the variation of pupil diameter over time for different, constant levels of illumination. This effect is most often associated with the pulsation of blood in its blood vessels. Some authors have carried out studies of pupil size along with other signals. That led to a description of the relationship between fluctuations in pupil diameter and heart rate $^{16}$ or fluctuations in pupil diameter and respiratory fluctuations. ${ }^{17}$ The size of the pupil also changes as a result of the accommodation reflex.

On the other hand, apart from neovascular glaucoma, no changes in iris vasculature have been reported. The effect of a pulse wave in the peripheral tissues has been described several times in the past. Pulse wave is the spread of arterial deformation, which is caused by a change in blood volume that is pushed from the heart with every heartbeat. It propagates at a speed of $5-8 \mathrm{~m} / \mathrm{s}$, while the blood flow rate is around $0.5 \mathrm{~m} / \mathrm{s}$.

Dynamic changes in pupil size have been used to objectively measure the influence of chronic stress. In a study by Al Abdi et al., ${ }^{18}$ a device for physiological measurements was developed to diagnose chronic stress, based on blunted reactivity of the autonomic nervous system to cognitive load. The authors documented blunted cognitive-load-induced changes in pulse wave amplitude, galvanic skin response and pupil diameter in stressed subjects in comparison with non-stressed ones. Preliminary results have demonstrated the ability of these methods to objectively detect chronic stress. The most interesting aspect is the work by Al Abdi et al. who proved that pupil diameter is under the control of the autonomic system and that a degradation of the autonomic system is strongly represented in the ability of the pupil to change its geometry.

We hypothesize that the impaired autonomic regulation of pupil size in glaucoma and glaucoma suspects is an early sign of this disease and, therefore, the vasculature of the iris has an impact on short-term changes in pupil shape. This is caused by pulse wave propagation to distant tissues - when the autonomic innervation (sympathetic and parasympathetic pathways) is impaired, iris muscles atrophy. Under these conditions, the autonomic regulation of systemic circulation overcomes local regulation of pupil size represented by the neuromuscular unit in the iris to the point at which it can be detected (Fig. 1).

To the best of our knowledge, this is the first study that addresses the correlation between peripheral arterial pulsation and pupil size and shape changes in early glaucoma detection. 


\section{Material and methods}

\section{Study design}

This was an investigator-initiated, single-center prospective cohort study conducted at the Department of Optics and Photonics, Wroclaw University of Science and Technology, and Department of Ophthalmology, Wroclaw Medical University, Poland. The project was approved by the Ethics Committee of the Wroclaw Medical University (approval No. KB 246/2017) and it adhered to the tenets of the Declaration of Helsinki.

\section{Study population}

Patients with diagnosed glaucoma or glaucoma suspects were prospectively enrolled in the study. The included patients were those who were diagnosed with glaucoma and glaucoma suspects who converted to glaucoma within 5 years from inclusion. Detailed inclusion and exclusion criteria can be found in Table 1. All patients underwent a complete ophthalmological examination, including slitlamp biomicroscopy, fundoscopy and gonioscopy, as well as visual field testing using a standard white-on-white visual field (Humphrey Field Analyzer (HFA) II 750; 24-2 Swedish interactive threshold algorithm; Carl Zeiss Meditec, Dublin, USA) and RNFL thickness measurement using a Spectralis OCT (Heidelberg Engineering, Heidelberg, Germany). Additionally, the retinal ganglion cell complex (GCC) was assessed using an RtVue AngioOCT (Optovue, Fremont, USA). The following baseline data were recorded: age, gender, best-corrected visual acuity, preoperative IOP with Goldmann applanation tonometry, and medication score.

The study population was divided into 3 groups: 25 eyes in the glaucoma suspect group (GSG), 22 eyes in the glaucoma group (GG) and 28 eyes in the control group (CG). Subjects were fully informed of the purpose of the study and about all the procedures and their requirements. Informed consent was obtained from the patients before any measurements were taken.

\section{Experimental measurements}

The patient's head was stabilized in a rigid head rest, designed and built by the researchers. The patient was asked to gaze at the motionless fixation point and to abstain from blinking for up to $14 \mathrm{~s}$. Thanks to this eye fixation, an assumption about the constant impact of corneal refraction on the pupil image was made. If possible, the examination was carried out on both eyes of each patient. However, the study included people during the post-operative period (cataract) or who were already monocular; hence, it was not possible to examine both eyes of each person in each case.

The main component of the system for recording images of the human pupil was a fast CMOS camera (AOS S-PRI; AOS Technologies AG, Baden, Switzerland). ${ }^{19}$ Together with the photo lens, it ensures an optimal image size of the pupil on the image sensor $(800 \times 600$ pixels $)$. Acceptable contrast was obtained by means of retro-illumination registered by the camera. To preclude the pupils responding to the lighting system, infrared illumination was used. Peripheral arterial pulsation was synchronously recorded with the recording of video sequences of the pupils with the use of an MLT1010 pulsometer attached to the subject's finger (AD Instruments, Sydney, Australia). The sequences were recorded at a speed of $200 \mathrm{fps}$ and the peripheral arterial pulsation signal had a rate of $200 \mathrm{~Hz}$.

The video sequence was divided into single frames (up to 2,700 frames), and each of them was numerically analyzed with the use of Matlab R2015b (The MathWorks, Inc., Natick, USA). Border points of the pupil were selected automatically using an edge detection procedure (Fig. 2). Due to differences in magnification of the pupil, the pupil size was calibrated for each sequence. The pupil image was treated as a filled plane figure. The shape of the pupil was approximated with the best fitting ellipse, understood as the ellipse with the same second moments of inertia as the pupil shape (Fig. 2). The area of the pupil approximated by the ellipse $(S)$ and the length of its major and minor semi-axes ( $a$ and $b$, respectively) were determined. Based on the major and minor semi-axes, the eccentricity $(\varepsilon)$ was determined with the use of the following equation:

$$
\varepsilon=\sqrt{\frac{a^{2}-b^{2}}{a^{2}}}
$$

Table 1. Inclusion and exclusion criteria

\begin{tabular}{|c|c|}
\hline Inclusion criteria & Exclusion criteria \\
\hline $\begin{array}{l}\text { 1. } 18 \text { years of age or older. } \\
\text { 2. Diagnosis of glaucoma or glaucoma suspect done previously and re- } \\
\text { assessed during screening by ophthalmologist. } \\
\text { 3. Medicated } I O P \geq 15 \leq 35 \mathrm{~mm} \mathrm{Hg} \text {. } \\
\text { 4. Subjects in glaucoma group taking } 1-5 \mathrm{IOP} \text {-lowering medications. } \\
\text { 5. Conversion to glaucoma diagnosed within a } 5 \text {-year period (from } \\
\text { the time of recordings) in glaucoma suspect group. } \\
\text { 6. Signed informed consent. }\end{array}$ & $\begin{array}{l}\text { 1. Active inflammation (e.g., blepharitis, conjunctivitis, keratitis, or uveitis). } \\
\text { 2. Active iris neovascularization or neovascularization of the iris within } \\
\text { the previous } 6 \text { months. } \\
\text { 3. Anterior chamber intraocular lens. } \\
\text { 4. Presence of intraocular silicone oil. } \\
\text { 5. Vitreous present in the anterior chamber. } \\
\text { 6. Impaired episcleral venous drainage (e.g., Sturge-Weber } \\
\text { or nanophthalmos or other evidence of elevated venous pressure). } \\
\text { 7. History of dermatological keloid formation. } \\
\text { 8. Previous photorefractive keratectomy (PRK). }\end{array}$ \\
\hline
\end{tabular}




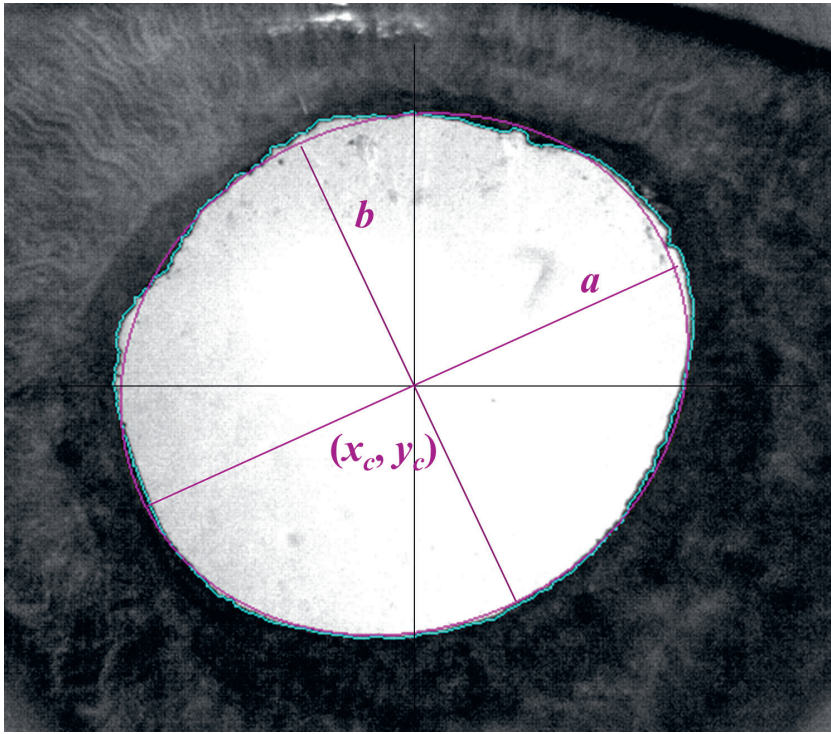

Fig. 2. Exemplary pupillary edge of iris (light blue corrugated line) with the best fitted ellipse (purple line) and its parameters: $\left(x_{c}, y_{c}\right)$-center of the ellipse, $a$ - major and $b$ - minor semi-axis

The temporal variability of the parameters $S$ and $\varepsilon$, as well as the peripheral arterial pulsation $(p)$, was subjected to further analysis.

\section{Numerical analysis}

All analyzed signals were subjected to spectral analysis using fast Fourier transform (FFT) and correlation analysis with the use of the coherence function between pairs of recorded signals. Values of the coherence function are always in the range of 0 to 1 . Higher values of the coherence function between 2 signals means higher spectral correlation between the signals.

Two of the signals, $S$ and $p$, analyzed over time for exemplary sequence, are shown in Fig. 3. Their basic registered
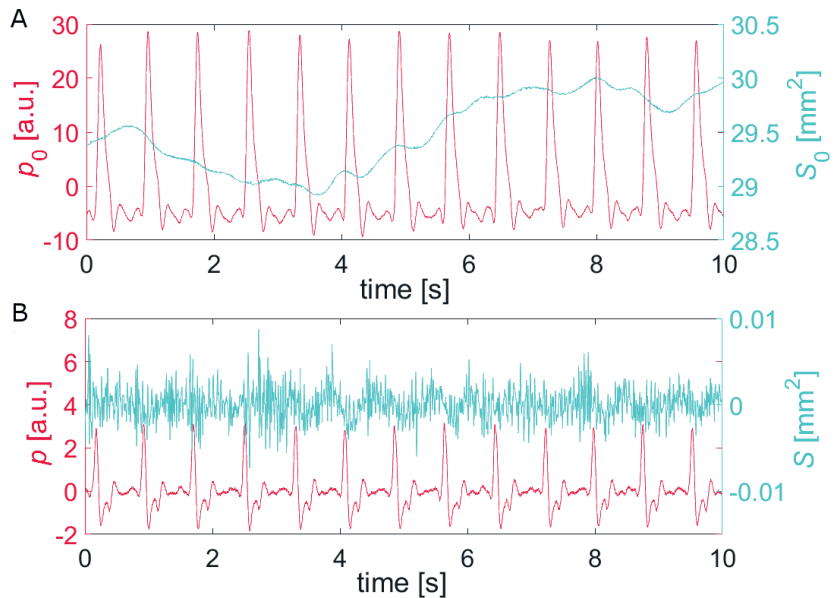

Fig. 3. Examples of basic signals $p$ and $S A$ ) in their basic registered form $p_{0}$ and $S_{0}$ and $B$ ) differentiated signals for further analysis $p$ and $S$

$p$ - the peripheral arterial pulsation signal; $S$ - the area of the eye pupil; $p_{0}$ - basic registered peripheral arterial pulsation signal; $S_{0}$ - basic registered area of the eye pupil. form, marked with subscript 0 - that is, $p_{0}$ and $S_{0}$ - is presented in Fig. 3A. Figure 3B shows their differentiated forms for further analysis, denoted as $S$ and $p$, respectively, in order to better visualize the effect of higher frequencies.

Periodograms (marked as PFT) of the modified signals calculated by the use of FFT are presented in Fig. 4A. Peaks in the PFT periodograms stand for characteristic frequencies present in the signal. However, the components with that frequency may appear at different times in the analyzed signals. The coherence function allows frequencies present in both signals at the same time to be located with the use of Welch's overlapped averaged periodogram. ${ }^{20}$ The coherence function between the 2 modified signals presented in Fig. $3 \mathrm{~B}^{21}$ is shown in Fig. $4 \mathrm{~B}$. To determine and quantitatively compare correlations between 2 analyzed signals, the average value of the coherence function above 0.5 was determined. This parameter, called coherence level, was defined as:

$$
\operatorname{lev} C_{z_{1} z_{2}}=\frac{\sum_{i=1}^{j} C_{z_{1} z_{2}}^{*}(i)}{j}
$$

where

$$
C_{z_{1} z_{2}}^{*}(i)=\left\{\begin{array}{cl}
C_{z_{1} z_{2}}(i) & \text { for } C_{z_{1} z_{2}}(i) \geq 0.5 \\
0 & \text { for } C_{z_{1} z_{2}}(i)<0.5
\end{array}\right.
$$

and where $z_{1}, z_{2}$ represent pairs of parameters and $i$ denotes the next values $i=1,2, \ldots, j$ of frequencies in the range of $0-40 \mathrm{~Hz}$ for which the coherence value has been determined. A graphical interpretation of the coherence level is shown in Fig. 4B, though for better visualization the graph presents only values up to $20 \mathrm{~Hz}$. The points above the red line at the level of 0.5 were taken into consideration when determining the value of the coherence level. The coherence level parameter is with some approximation proportional
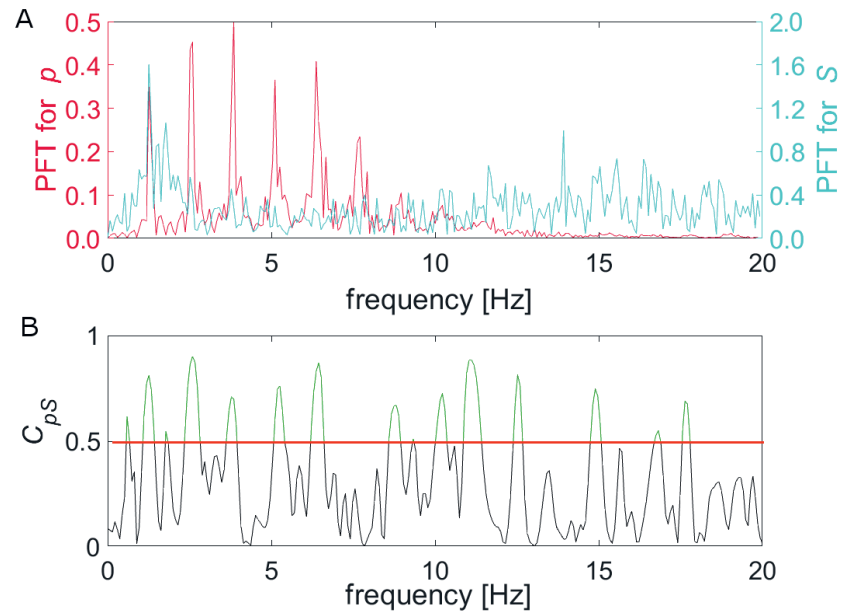

Fig. 4. A) PFT periodograms for $p$ and $S$ signals and B) coherence function with marked values to calculate coherence level

$p$ - peripheral arterial pulsation signal; $S$ - area of the eye pupil; $C_{p s}$ - coherence function between $p$ and $S$; lev $C_{p s}$ - coherence level. 
to the area below the coherence function and above the line indicating the coherence level equal to 0.5 . It was assumed that the coherence values $C_{z_{1} z_{2}}$ below 0.5 do not indicate a significant correlation between the signals analyzed. Thus, the coherence level parameter describes the characteristic coherence level in the range $0-40 \mathrm{~Hz}$.

\section{Outcome measures}

The primary outcome measure was the coherence level between the systemic pulsation signal and the change in the area of the pupil. Secondary outcome measures included correlations between RNFL and GCC thicknesses and the coherence level, as well as correlations between the coherence level and the change in RNFL and GCC thicknesses over the 5-year follow-up period.

\section{Statistical analysis}

Almost each participant of all 3 groups -GG, GSG and CG - was recorded at least twice; analysis of variance (ANOVA) factorial analyses were performed to determine the existence of any connection between the $1^{\text {st }}$ and $2^{\text {nd }}$ recordings. Descriptive statistics for each parameter, such as RNFL, GCC and their change over the years, and the coherence levels, including median and $95 \%$ confidence intervals $(95 \% \mathrm{CI})$ for the median, were used. The crosstabs with relative risk and odds ratio (OR) analysis using $z$-scores were conducted for all parameters. Comparisons of 2 independent samples were performed with the Mann-Whitney test and, for multiple independent samples, an ANOVA Kruskal-Wallis test was used. In all analyses, statistical significance was recognized if $\mathrm{p}<0.05$ for primary and $\mathrm{p}<0.001$ for secondary outcome measures. The analysis of correlations was conducted by means of Spearman's $\mathrm{R}$ test $(\mathrm{p}<0.001)$. In the next step, the Stepwise Model Builder from STATISTICA v. 13.3 (StatSoft Inc., Tulsa, USA) for linear regression was used, in which the next variable was added only if the overall fitness of the model was improved with a significance level of $\mathrm{p}<0.05$. Some of the abovementioned calculations were made in Matlab R2015b (The MathWorks, Inc., Natick, USA) as well.

\section{Results}

The characteristics of the study population are described in Table 2. Figure 5 shows box-plot charts for 3 of the values of the coherence level: $\operatorname{lev} C_{p S}, \operatorname{lev} C_{p \varepsilon}$ and $\operatorname{lev} C_{p \theta}$. Please note that the vertical axis of Fig. $5 \mathrm{C}$ is expressed in different units than the other 2 graphs.

Statistical analysis revealed a significant difference between CG and both the GSG and GG for all 3 coherence level parameters (Fig. 5). The median value of lev $C_{p S}$ in the GG (0.102) was much higher than that of the CG (0.038; $\mathrm{p}<0.001)$. Moreover, the GSG had a higher median value (0.086) than the CG $(\mathrm{p}<0.001)$. The median values of lev $C_{p \varepsilon}$

Table 2. Characteristics of subjects in the study groups

\begin{tabular}{|l|c|c|c|}
\multicolumn{1}{|c|}{ Parameter } & GSG & GG & CG \\
\hline Number of eyes measured & 25 & 22 & 28 \\
\hline Age median (IQR) [years] & $64(35-72)$ & $63(53-70)$ & $31(24-41)$ \\
\hline Female/male ratio & $16: 9$ & $15: 7$ & $15: 13$ \\
\hline
\end{tabular}

GSG - glaucoma suspect group; GG - glaucoma group; CG - control group; IQR - interquartile range.
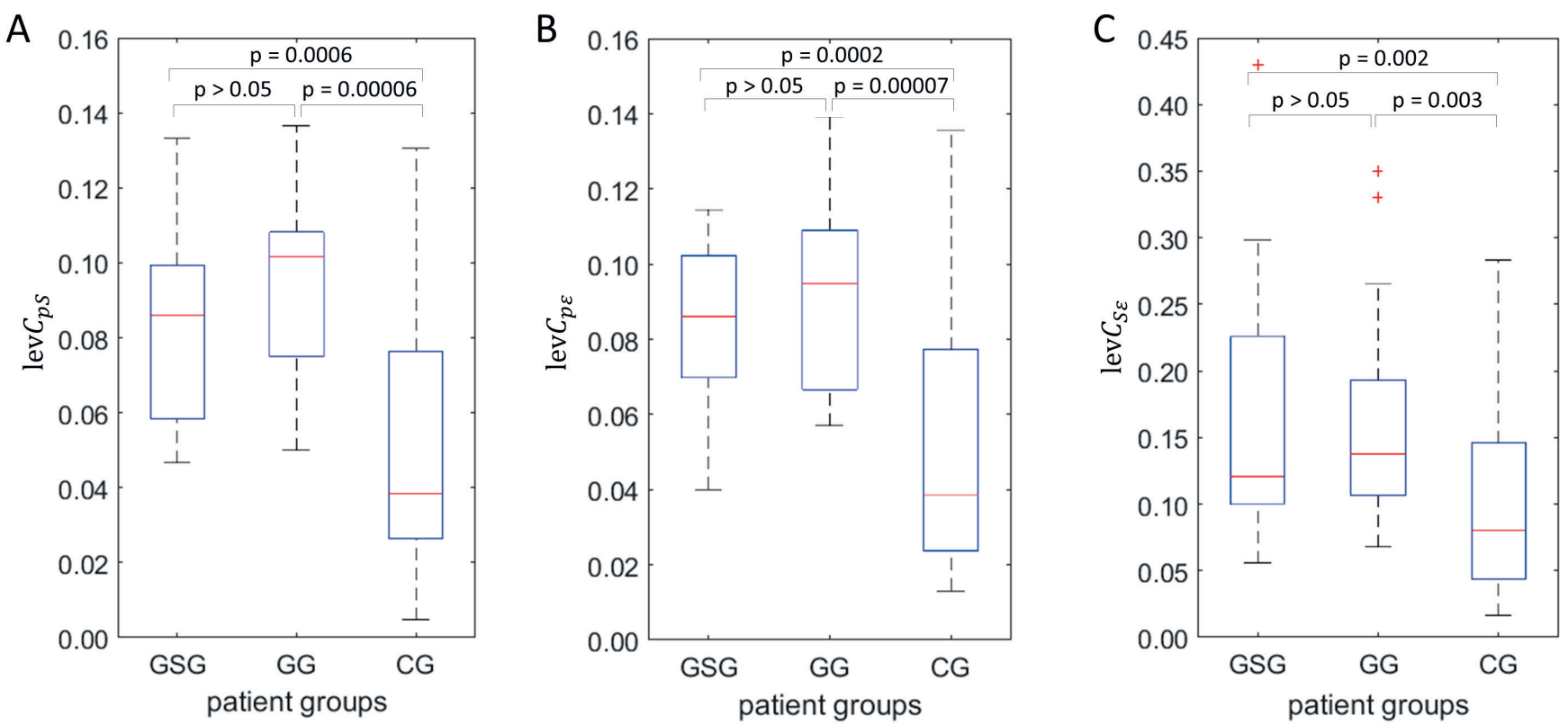

Fig. 5. Box-plot for lev ( calculated for pair of signals: A) $p$ and S, B) $p$ and $\varepsilon$ and C) $S$ and $\varepsilon$ for analyzed patient groups

GG - glaucoma group; GSG - glaucoma suspect group; CG - control group; IQR - interquartile range; HR - heart rate; levC - coherence level; $p$ - peripheral arterial pulsation; $S$ - area of the pupil; $\varepsilon$ - eccentricity; ${ }^{*} p<0.05 ;{ }^{* *} p<0.01 ;{ }^{* * *} p<0.001$. 
Table 3. Summary of differences between GG and GSG groups (change in RFNL and GCC thickness were observed during follow-up period)

\begin{tabular}{|c|c|c|c|}
\hline $\begin{array}{c}\text { Parameter median } \\
\text { and IQR }\end{array}$ & $\begin{array}{c}\mathrm{GG} \\
(\mathrm{n}=22)\end{array}$ & $\begin{array}{c}\text { GSG } \\
(n=25)\end{array}$ & $p$-value \\
\hline Observation time [years] & $\begin{array}{c}4 \\
(3.5-5.0)\end{array}$ & $\begin{array}{c}3 \\
(2.56-4.10)\end{array}$ & $p>0.05$ \\
\hline RNFL $[\mu \mathrm{m}]$ & $\begin{array}{c}70 \\
(56-86)\end{array}$ & $\begin{array}{c}95 \\
(86-97)\end{array}$ & 0.001384 \\
\hline$\triangle \mathrm{RNFL}[\mu \mathrm{m}]$ & $\begin{array}{c}4.6 \\
(4.2-5.2)\end{array}$ & $\begin{array}{c}6.3 \\
(5.4-7.1)\end{array}$ & 0.000006 \\
\hline$\triangle R_{N F} L^{y}[\mu m / y e a r]$ & $\begin{array}{c}0.94 \\
(0.84-1.20)\end{array}$ & $\begin{array}{c}0.66 \\
(0.54-0.71)\end{array}$ & 0.000010 \\
\hline $\mathrm{GCC}[\mu \mathrm{m}]$ & $\begin{array}{c}81 \\
(72-88)\end{array}$ & $\begin{array}{c}91 \\
(88-98)\end{array}$ & 0.001026 \\
\hline$\Delta \mathrm{GCC}[\mu \mathrm{m}]$ & $\begin{array}{c}4.45 \\
(4.1-5.1)\end{array}$ & $\begin{array}{c}7.0 \\
(5.2-8.1)\end{array}$ & 0.000007 \\
\hline$\Delta \mathrm{GCC}^{\mathrm{y}}[\mu \mathrm{m} / \mathrm{year}]$ & $\begin{array}{c}1.26 \\
(0.95-1.39)\end{array}$ & $\begin{array}{c}1.34 \\
(1.05-1.57)\end{array}$ & 0.000021 \\
\hline
\end{tabular}

IQR - interquartile range; GG - glaucoma group; GSG - glaucoma suspect group; RNFL - retinal nerve fiber layer; $\triangle R N F L$ - loss of retinal nerve fiber layer; $\triangle \mathrm{RNFL}$ - loss of retinal nerve fiber layer per year; GCC - ganglion cell complex; $\triangle \mathrm{GCC}$ - loss of ganglion cell complex; $\triangle \mathrm{GCC}$ - loss of ganglion cell complex per year.

for both the GSG (0.086) and GG (0.095) were higher than that of the CG $(0.038 ; \mathrm{p}<0.001)$. The differences in median values of lev $C_{p \varepsilon}$ for both the GSG (0.121) and GG (0.138) were significantly higher in comparison to the CG (0.080; $<<0.01)$.

Clinical data of RNFL thickness and GCC thickness measured at the initial examination and reviewed during the 5-year observational period are summarized in Table 3. The loss of retinal nerve fiber layer $(\triangle \mathrm{RNFL})$ calculated for each measured eye separately represents the difference between RNFL thickness values at 2 points in time, when the pupil was measured and after up to 5 years, which was when the GSG patients had converted to glaucoma.
In regards to the GG patients, the median observation time was 4 years $(95 \% \mathrm{CI}=3.5-5.0)$. The value $\triangle \mathrm{RNFL}^{\mathrm{y}}$ represents $\triangle$ RNFL per year. The same applies to GCC, the loss of ganglion cell complex $(\triangle \mathrm{GCC})$ and the yearly loss of ganglion cell complex $(\triangle \mathrm{GCC})$.

During the follow-up period, there was a larger decrease in RNFL and GCC thickness in the GSG than in the GG $(p<0.05)$. When it comes to the GSG, it should be stressed that the observation period was different for each patient. If a patient classified as GSG developed a visual field defect, the researcher was able to diagnose glaucoma and treatment was introduced; therefore, a change in RNFL and GCC thickness per year was calculated in a different timeframe for each patient. The median time that was required to detect conversion towards glaucoma was 3 years (95\% CI $=2.56-4.10)$. Additionally, it should be highlighted that this is the first study to describe the change in GCC thickness in GSG patients. Interestingly, when the coherence levels were compared to the clinical data, there were strong correlations in GG and GSG. It is worth mentioning that the correlations between the coherence levels were stronger in the GSG (e.g., $\triangle$ RNFL thickness $(\mathrm{r}=0.732 ; \mathrm{p}<0.0001)$ or $\triangle \mathrm{GCC}$ thickness $(\mathrm{r}=0.6491$; $\mathrm{p}<0.0001$ ), while in the GG it was not statistically significant). The correlation between lev $C$ and RNFL thickness $(\mathrm{r}=-0.7539 ; \mathrm{p}<0.0001)$ and GCC thickness $(\mathrm{r}=-0.6509$; $\mathrm{p}=0.001)$ in the GG is presented in Fig. 6A,B. This progression was not observed in the GG during follow-up ( $\triangle$ RNFL and $\triangle G C C$ thicknesses were not statistically significant). Additionally, as the patients from the GSG developed visual field defects during the observation period which resulted in a change of diagnosis toward glaucoma, we therefore focused on presenting the changes in RNFL and GCC thicknesses in the GSG during this time (Fig. 6C,D).

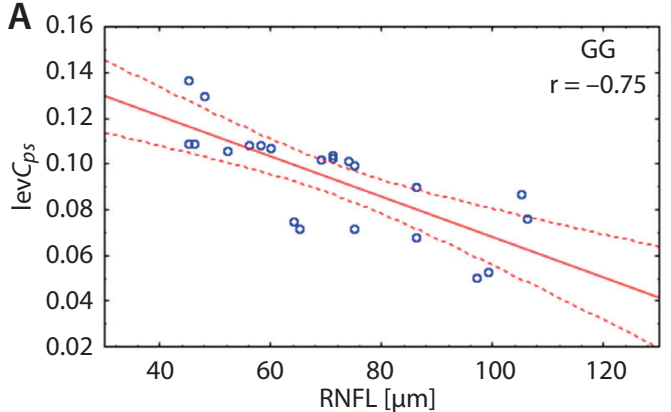

C

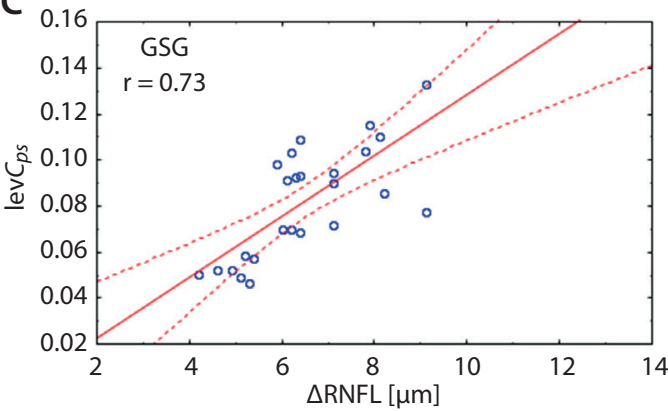

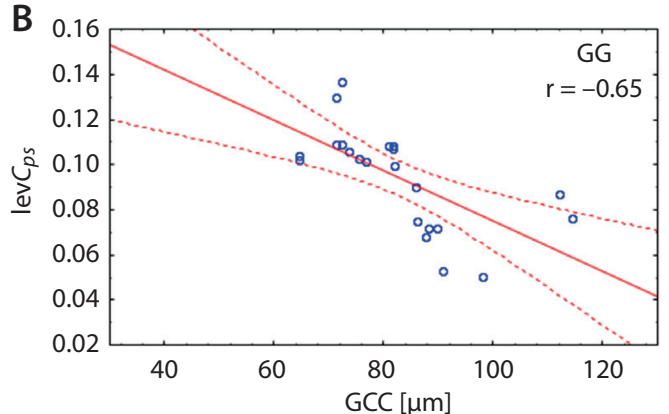

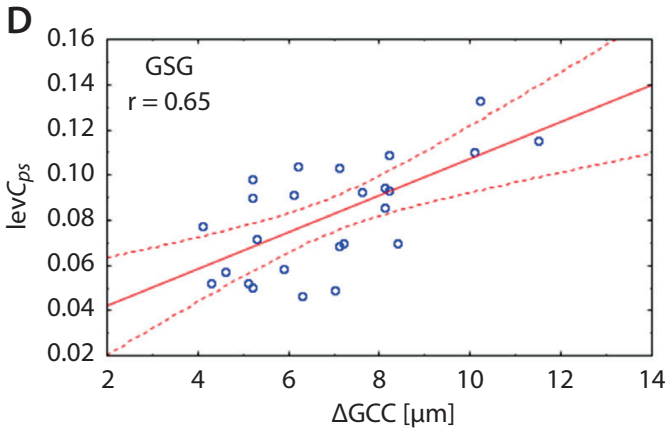

Fig. 6. Correlations between levC and different clinical indicator of progression in glaucomatous ( $A$ and $B$ ) and glaucoma suspect group (C and D)

lev C - coherence level; $p$ - peripheral arterial pulsation; $S$ - area of the pupil; RNFL - retinal nerve fiber layer; $\triangle \mathrm{RNFL}$ - loss of retinal nerve fiber layer; GCC - ganglion cell complex; $\triangle \mathrm{GCC}$ - loss of ganglion cell complex; GG - glaucoma group; GSG - glaucoma suspect group. 


\section{Discussion}

Taking into account the neuromuscular regulation of pupil size in a normal iris, the influence of peripheral arterial pulsation on the pupillary margin of the iris will be minimal. The situation is different in glaucomatous eyes, where the non-image-forming pathway that controls pupil size is impaired due to the loss of ipRGCs (Fig. 1). In glaucoma, the pupillary margin is affected by peripheral arterial pulsation, thus periodic changes in pupil size and shape that are consistent with the heartbeat can be found in those patients.

While decreased pupil constriction in response to red or blue stimuli was reported in the glaucoma group, in the ocular hypertension $(\mathrm{OH})$ group, similar changes were not found by Kelbsch et al. It is worth mentioning that in our study, during the 5-year follow-up period all but 1 patient from the glaucoma suspect group converted to glaucoma, while in the research published by Kelbsch et al. no such data is available. ${ }^{10}$ Additionally, the results of the Ocular Hypertension Treatment Study (OHTS) provide clinically important information on the rate of conversion from $\mathrm{OH}$ to glaucoma during the 5-year follow-up period. Quoting the OHTS, "the cumulative probability of developing primary open angle glaucoma (POAG) was reduced by $60 \%$ among participants randomized to receive typical ocular hypotensive medication compared with those randomized to observation (hazard ratio $=0.40$; $95 \% \mathrm{CI}=0.27-0.59$ ). At 60 months, the cumulative probability of developing POAG was $4.4 \%$ in the medication group and $9.5 \%$ in the observation group". ${ }^{22}$ Assuming that the German group included 16 patients with $\mathrm{OH}$, only 1 or 2 of them would convert to glaucoma over the 5 years of follow-up if left untreated.

In a paper by Calcagni et al., ${ }^{23}$ heart rate, blood pressure, respiration, peripheral blood flow, and pupil diameter were simultaneously measured in constant light conditions in order to determine whether there is any correlation between those signals, particularly whether fluctuations in blood pressure contribute to spontaneous pupil fluctuations. It was noted that stimulation of the carotid baroreceptors induced pupil size fluctuations. On the other hand, taking into account that the sympathetic pathway of non-image-forming input signals from ipRGCs are led through the carotid plexus, it is likely that carotid sinus baroreceptors are stimulated at the same time as the carotid plexus axons from non-image-forming homonymous hemiretinal signals. Due to this probability, it is an extremely difficult task to distinguish between those 2 stimulations.

In the current study, we report on 5 years of follow-up observation of changes in RNFL and GCC thickness. In the GG, the rate of thickness change $\triangle R_{N F L}{ }^{y}$ was $0.94 \mu \mathrm{m} /$ year, while for $\Delta \mathrm{GCC}^{\mathrm{y}}$ it was $0.66 \mu \mathrm{m} /$ year. However, in the GSG, both measurements were higher: $1.26 \mu \mathrm{m} /$ year and $1.34 \mu \mathrm{m} /$ year for $\Delta \mathrm{RNFL}^{\mathrm{y}}$ and $\Delta \mathrm{GCC}^{\mathrm{y}}$ thicknesses, respectively. Similar results for glaucomatous patients were presented by Hammel et al. In their work, glaucomatous eyes were characterized by a mean rate of global circumpapillary $\triangle R_{N F L}$ thickness change of $-0.98 \mu \mathrm{m} /$ year and a normalized global circumpapillary $\triangle \mathrm{RNFL}^{\mathrm{y}}$ change of $-1.7 \%$ /year. Changes in RNFL thickness occurred significantly faster than the average macular GCC change $(-0.57 \mu \mathrm{m} /$ year $)$ and normalized macular GCC change (-1.3\%/year). ${ }^{24}$ In a paper by Kim et al., RNFL and GCC thicknesses were observed over a 3-year period and the rates were estimated to be on the level of $-1.23 \mu \mathrm{m} /$ year and $1.53 \mu \mathrm{m} /$ year, respectively. ${ }^{25}$ Additionally, the temporal relationship between GCC loss and RNFL loss in glaucomatous patients was confirmed. ${ }^{25,26}$ It is important to mention that in our work, we also observed a patient who was considered a glaucoma suspect at the beginning of the study and who converted to glaucoma during the follow-up period. In the GSG, the RNFL and GCC losses were greater than in the GG; however, this patient was not treated prior to their conversion. In this regard, interesting studies were published by Sehi et al. and Miki et al. In their work, Sehi et al. observed 310 glaucoma suspects and pre-perimetic glaucoma patients for over 3.3 years. In 89 eyes, visual field progression was found, and RNFL progression was reported in 101 eyes. Miki et al. compared 454 eyes of 294 glaucoma suspects during a 2.2-year follow-up period. The estimated mean rate of global RNFL loss was significantly faster in eyes developing visual field defects compared with eyes that did not $(-2.02 \mu \mathrm{m} /$ year vs $-0.82 \mu \mathrm{m} /$ year; $\mathrm{p}<0.001) .{ }^{27,28}$ We found slightly less deterioration in RNFL thickness than has been previously presented, though our study group was smaller and we introduced treatment with 2 anti-glaucoma drugs at once. This approach may play an important role in the early inhibition of GCC and RNFL loss.

This is the first time the coherence level between peripheral arterial pulsation and change in pupil geometry was measured. To the best of our knowledge, this is the first attempt to relate changes in the neuronal signaling pathways in glaucoma to the vascular-dependent changes of pupil geometry. The findings suggest that this approach could be used to define which glaucoma suspect patients have alterations in the autoregulation of pupil geometry which increase their likelihood of converting to glaucoma. This alternate autoregulation of pupil geometry might be due to autonomic system impairment in the eye, but this hypothesis needs to be examined further. In the future, the methodology presented by our group can open up a wide range of new approaches to glaucoma neurodegeneration. Further research is still needed, including electrophysiological tests. It is worth mentioning that the measurement is relatively brief $-12 \mathrm{~s}$. Additionally, during recording no provocative tests, such as flashes of red or blue light under specific conditions, were required to evoke ipRGC potentials. 


\section{ORCID iDs}

Marta Anna Szmigiel (D) https://orcid.org/0000-0002-2823-4859 Joanna Wiktoria Przeździecka-Dołyk

(iD) https://orcid.org/0000-0002-1099-4876

Jacek Olszewski (D) https://orcid.org/0000-0002-8978-6059

Henryk Kasprzak (1) https://orcid.org/0000-0002-4241-1954

\section{References}

1. Parihar JKS. Glaucoma: The 'black hole' of irreversible blindness. Med J Armed Forces India. 2016;72(1):3-4.

2. World Health Organization. Vision impairment and blindness. https:// www.who.int/news-room/fact-sheets/detail/blindness-and-visualimpairment. Accessed February 23, 2019.

3. Gracitelli CPB, Duque-Chica GL, Moura AL, et al. Relationship between daytime sleepiness and intrinsically photosensitive retinal ganglion cells in glaucomatous disease. J Ophthalmol. 2016;2016:5317371.

4. Kuze M, Morita T, Fukuda Y, Kondo M, Tsubota K, Ayaki M. Electrophysiological responses from intrinsically photosensitive retinal ganglion cells are diminished in glaucoma patients. J Optom. 2017;10(4): 226-232.

5. Gracitelli CPB, Duque-Chica GL, Moura AL, et al. A positive association between intrinsically photosensitive retinal ganglion cells and retinal nerve fiber layer thinning in glaucoma. Invest Ophthalmol Vis Sci. 2014;55(12):7997-8005.

6. Hattar S, Liao HW, Takao M, Berson DM, Yau KW. Melanopsin-containing retinal ganglion cells: Architecture, projections, and intrinsic photosensitivity. Science. 2002;295(5557):1065-1070.

7. Berson DM, Dunn FA, Takao M. Phototransduction by retinal ganglion cells that set the circadian clock. Science. 2002;295(5557):1070-1073.

8. Rukmini AV, Milea D, Baskaran M, et al. Pupillary responses to highirradiance blue light correlate with glaucoma severity. Ophthalmology. 2015;122(9):1777-1785.

9. El-Danaf RN, Huberman AD. Characteristic patterns of dendritic remodeling in early-stage glaucoma: Evidence from genetically identified retinal ganglion cell types. J Neurosci. 2015;35(6):2329-2343.

10. Kelbsch C, Maeda F, Strasser T, et al. Pupillary responses driven by ipRGCs and classical photoreceptors are impaired in glaucoma. Graefes Arch Clin Exp Ophthalmol. 2016;254(7):1361-1370.

11. Kankipati L, Girkin CA, Gamlin PD. The post-illumination pupil response is reduced in glaucoma patients. Invest Ophthalmol Vis Sci. 2011;52(5):2287-2292.

12. Wyatt HJ. The form of the human pupil. Vision Res. 1995;35(14): 2021-2036.

13. Nowak W, Hachoł A, Kasprzak H. Time-frequency analysis of spontaneous fluctuation of the pupil size of the human eye. Optica Applicata. 2008;38(2):469-480.

14. Gooley JJ, Ho Mien I, St Hilaire MA, et al. Melanopsin and rod-cone photoreceptors play different roles in mediating pupillary light responses during exposure to continuous light in humans. J Neurosci. 2012;32(41):14242-14253.
15. Winn B, Whitaker D, Elliott DB, Phillips NJ. Factors affecting lightadapted pupil size in normal human subjects. Invest Ophthalmol Vis Sci. 1994;35(3):1132-1137.

16. Yoshida H, Mizuta H, Gouhara T, Suzuki Y, Yana K, Okuyama F. Statistical properties of simultaneously recorded fluctuations in pupil diameter and heart rate. Proceedings of $17^{\text {th }}$ International Conference of the Engineering in Medicine and Biology Society (IEEE 1995), Montreal, Canada:165-166.

17. Yoshida, H, Yana K, Okuyama F, Tokoro T. Time-varying properties of respiratory fluctuations in pupil diameter of human eyes. Methods InfMed. 1994;33(1):46-48.

18. Al Abdi RM, Alhitary AE, Abdul Hay EW, Al-Bashir AK. Objective detection of chronic stress using physiological parameters. Med Biol Eng Comput. 2018;56(12):2273-2286.

19. Szmigiel M, Kasprzak H. Distribution of parameters of elliptic approximation of the human pupil. Optica Aplicata. 2015;45(1):41-50.

20. Welch $P$. The use of fast Fourier transform for the estimation of power spectra: A method based on time averaging over short, modified periodograms. IEEE Transactions on Audio and Electroacoustics. 1967; 15(2):70-73.

21. Szmigiel MA, Kasprzak H, Klysik A. Dependences between kinetics of the human eye pupil and blood pulsation. In: Applications of Digital Image Processing XXXIX, International Society for Optics and Photonics, August 29 - September 1, 2016, San Diego, USA, art. 99712V, s. 1-9; SPIE Proceedings Series, vol. 9971

22. Gordon MO, Beiser JA, Brandt JD, et al. The Ocular Hypertension Treatment Study: Baseline factors that predict the onset of primary open-angle glaucoma. Arch Ophthalmol. 2002;120(6):714-720.

23. Calcagini G, Censi F, Lino S, Cerutti S. Spontaneous fluctuations of human pupil reflect central autonomic rhythms. Methods Inf Med. 2000;39(2):142-145.

24. Hammel N, Belghith A, Weinreb RN, Medeiros FA, Mendoza N, Zangwill LM. Comparing the rates of retinal nerve fiber layer and ganglion cell-inner plexiform layer loss in healthy eyes and in glaucoma eyes. Am J Ophthalmol. 2017;178:38-50.

25. Kim YK, Ha A, Na KI, Kim HJ, Jeoung JW, Park KH. Temporal relation between macular ganglion cell-inner plexiform layer loss and peripapillary retinal nerve fiber layer loss in glaucoma. Ophthalmology. 2017;124(7):1056-1064.

26. Lee WJ, Kim YK, Park KH, Jeoung JW. Trend-based analysis of ganglion cell-inner plexiform layer thickness changes on optical coherence tomography in glaucoma progression. Ophthalmology. 2017;124(9): 1383-1391.

27. Miki A, Medeiros FA, Weinreb RN, et al. Rates of retinal nerve fiber layer thinning in glaucoma suspect eyes. Ophthalmology. 2014;121(7): 1350-1358.

28. Sehi M, Zhang X, Greenfield DS, et al. Retinal nerve fiber layer atrophy is associated with visual field loss over time in glaucoma suspect and glaucomatous eyes. Am J Ophthalmol. 2013;155(1):73-82.e1. 